\title{
Disentangling the Physical Contributions to the Electrical Resistance in Magnetic Domain Walls: A Multiscale Study
}

\author{
K. M. Seemann, ${ }^{1, *}$ F. Garcia-Sanchez, ${ }^{1}$ F. Kronast, ${ }^{2}$ J. Miguel, ${ }^{3}$ A. Kákay, ${ }^{1}$ C. M. Schneider, ${ }^{1}$ and R. Hertel ${ }^{4}$ \\ ${ }^{1}$ Peter Grünberg Institute, Forschungszentrum Jülich and Jülich-Aachen Research Alliance, D-52425 Jülich, Germany \\ ${ }^{2}$ Helmholtz-Zentrum Berlin für Materialien und Energie, Albert-Einstein-Strasse 15, Berlin, Germany \\ ${ }^{3}$ Diamond Light Source Ltd., Harwell Science and Innovation Campus, OX11 ODE Didcot, United Kingdom \\ ${ }^{4}$ Institut de Physique et Chimie des Matériaux de Strasbourg, Université de Strasbourg, CNRS UMR 7504, Strasbourg, France \\ F. Freimuth, Y. Mokrousov, and S. Blügel \\ Peter Grünberg Institute and Institute for Advanced Simulation, Forschungszentrum Jülich \\ and Jülich-Aachen Research Alliance, D-52425 Jülich, Germany
}

(Received 30 June 2011; published 13 February 2012)

\begin{abstract}
We analyze the origin of the electrical resistance arising in domain walls of perpendicularly magnetized materials by considering a superposition of anisotropic magnetoresistance and the resistance implied by the magnetization chirality. The domain wall profiles of $L 1_{0}$-FePd and $L 1_{0}$-FePt are determined by micromagnetic simulations based on which we perform first-principles calculations to quantify electron transport through the core and closure region of the walls. The wall resistance, being twice as high in $L 1_{0}$ - FePd than in $L 1_{0}-\mathrm{FePt}$, is found to be clearly dominated in both cases by a high gradient of magnetization rotation, which agrees well with experimental observations.
\end{abstract}

DOI: 10.1103/PhysRevLett.108.077201

PACS numbers: 75.60.Ch, 72.15.- $-\mathrm{v}, 75.25 .-\mathrm{j}$

Magnetic domain walls are fundamental structures in ferromagnets and have been the subject of intense research in the past years. The spin polarization of an electric current flowing in a ferromagnet and its interaction with the domain wall have been shown to be a new way of employing the spin degree of freedom of the electron. After the discovery of fundamental physical phenomena such as giant magnetoresistance [1] and tunneling magnetoresistance [2], which have revolutionized mass data storage technology, the effects of spin-polarized electrons acting on the magnetization of ferromagnetic material [3] have evolved rapidly into an interesting research field. The spintorque transfer effect is the main driving force behind the current-driven excitation of magnetization or the depinning and motion of domain walls along nanoscale ferromagnetic wires. It is explored in high frequency oscillators for microwave emission in vertical pillar-type devices of magnetic and nonmagnetic multilayers [4,5] but also in lateral devices where the magnetic domain wall (DW) has been proposed for advanced data storage [6] and logic processing [7].

While in-plane domain walls and inhomogeneous magnetization structures like vortices in $\mathrm{NiFe}$ alloys have been studied most [8,9], Bloch-type walls have been considered for high velocity current-driven wall motion experiments [10-12]. Such domain walls typically occur in materials with pronounced anisotropy, among which the epitaxial ferromagnets $L 1_{0}-\mathrm{FePd}$ and $L 1_{0}-\mathrm{FePt}$ are prominent examples. These two alloys exhibit a tetragonal crystal structure with uniaxial magnetic anisotropy and have strong spin-orbit interaction (SOI) stemming from heavy $4 d$ and
$5 d$ transition metals $\mathrm{Pd}$ and $\mathrm{Pt}$ [13]. The significant difference in SOI in $L 1_{0}-\mathrm{FePd}$ and $L 1_{0}-\mathrm{FePt}$ leads to a dramatic change in the physical origins of the transverse electron transport within the anomalous Hall effect [14]. This raises the question of the significance of SOI in electron transport in the longitudinal direction. On the other hand, the resistance due to the rotation of the magnetization also has to be taken into account. Quantitatively, domain wall magnetoresistance is usually described either by using ballistic models [15]—applied even to wide in-plane walls - or in the diffusive transport regime $[16,17]$. So far, a detailed analysis of DW resistance has not been performed for realistically shaped domain walls from first principles, while disentangling the different mechanisms is highly desirable for the manipulation of DW properties.

In this Letter, we report on a detailed multiscale analysis of the contributions to the electrical resistance in domain walls by combining the Kubo-Greenwood formalism with micromagnetically calculated domain wall structures which typically occur in perpendicularly magnetized materials. By augmenting the exact wall structure with the local electronic and transport properties, we are able to demonstrate that the SOI-driven anisotropic magnetoresistance (AMR) contribution to the DW resistance is small in comparison to the resistivity due to the rotation of the magnetization direction inside the wall, which dominates in both $L 1_{0}$-FePd and $L 1_{0}$-FePt. Overall, the DW resistivities in $\mathrm{FePt}$ and $\mathrm{FePd}$ calculated from first principles are in very good agreement with experimental values measured in these alloys. 
To gain a common basis for comparing the KuboGreenwood $a b$ initio approach to diffusive electron transport through a narrow Bloch domain wall with a four-probe transport experiment on a micron-sized structure, we consider experimentally determined areal resistivity values for one Bloch domain wall taken in zero field for $L 1_{0}-\mathrm{FePd}$ and $-\mathrm{FePt}$, respectively $[13,14]$. Figures 1(a) and 1(b) show the typical meandering domain structure of epitaxially deposited $L 1_{0}-\mathrm{FePd}$ and $-\mathrm{FePt}$ observed in $\mathrm{x}$-ray magnetic circular dichroismphotoelectron emission microscopy [18]. These experiments were performed at the UE49-PGM microfocus beam line of the BESSY II facility using synchrotron radiation monochromatized to the Fe- $L_{3}$ energy level. Magnetic contrast was obtained by reversing the helicity of the circularly polarized photons and depicting the asymmetry ratio.

In order to achieve a direct comparison with these experimental results, we computed the micromagnetic domain structure in both isoelectronic Fe alloys by using finite element micromagnetic simulations [19]. The static domain patterns and the detailed description of the domain wall structures were obtained by numerically integrating the Landau-Lifshitz-Gilbert equation with strong Gilbert damping. The material parameters of $\mathrm{FePt}$ - exchange stiffness $A=1.42 \times 10^{-11} \mathrm{~J} / \mathrm{m}$, uniaxial anisotropy constant $K=2.5 \times 10^{6} \mathrm{~J} / \mathrm{m}^{3}$, and saturation magnetization $\mu_{0} M_{s}=1.73 \mathrm{~T}$-and of $\mathrm{FePd}-A=1.6 \times 10^{-11} \mathrm{~J} / \mathrm{m}$, $K=1.5 \times 10^{6} \mathrm{~J} / \mathrm{m}^{3}$, and $\mu_{0} M_{s}=1.88 \mathrm{~T}$-used in micromagnetic calculations were determined by magnetometry experiments. The computational volume was discretized into tetrahedrons of about $1.3 \mathrm{~nm}$ edge length [20]. The equilibrium domain state was calculated in disks of $600 \mathrm{~nm}$ diameter and $30 \mathrm{~nm}$ thickness. The resulting domain pattern is representative for the demagnetized state observed in experiments as shown in Figs. 1(a) and 1(b) and insets. Red and blue areas mark domains of opposite
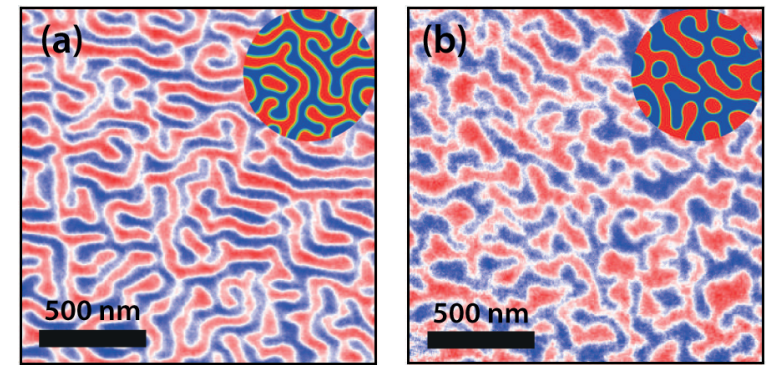

FIG. 1 (color online). X-ray magnetic circular dichroismphotoelectron emission microscopy asymmetry images of $L 1_{0}$-ordered $\mathrm{FePd}$ (a) and $\mathrm{FePt}$ (b) taken at the $\mathrm{Fe}$ $L_{3}$-absorption edge for the demagnetized domain state at room temperature and in zero magnetic field. The insets to the upper right corner show the result of micromagnetic simulations. Red and blue areas depict magnetic domains with magnetization orientated in opposite directions perpendicular to the film plane, while the fine lines in between domains illustrate Bloch domain walls. orientation of the perpendicularly magnetized films which are separated by narrow domain walls appearing as white lines.

We have also performed a detailed analysis of the structure of the domain walls. The cross section of approximately $10 \mathrm{~nm}$ narrow Bloch domain walls forming in films of $30 \mathrm{~nm}$ thickness exhibits a well-defined Bloch core at half thickness as illustrated in Fig. 2(a) for FePd and Fig. 2(b) for FePt. It is noteworthy that the Bloch core of the domain wall at half thickness of the film has very similar width in both materials, i.e., approximately $9 \mathrm{~nm}$ in $\mathrm{FePd}$ and $7 \mathrm{~nm}$ in $\mathrm{FePt}$, while the closure region towards the upper and lower edges of the cross section gives nearly $20 \mathrm{~nm}$ in the Pd alloy, twice the value of the Pt alloy.

The fraction of the wall volume in the domain pattern is calculated from the equilibrium domain structure of the $600 \mathrm{~nm}$ diameter disk and is summarized in Fig. 2(c) as a function of the absolute value of the maximal $M_{z}$ component, which in turn is evaluated for the wall profile in each material shown as insets in Fig. 2(c). Hence, we determine the interfacial resistivity for a single domain from magnetoresistance experiments [13] corrected for the fraction of
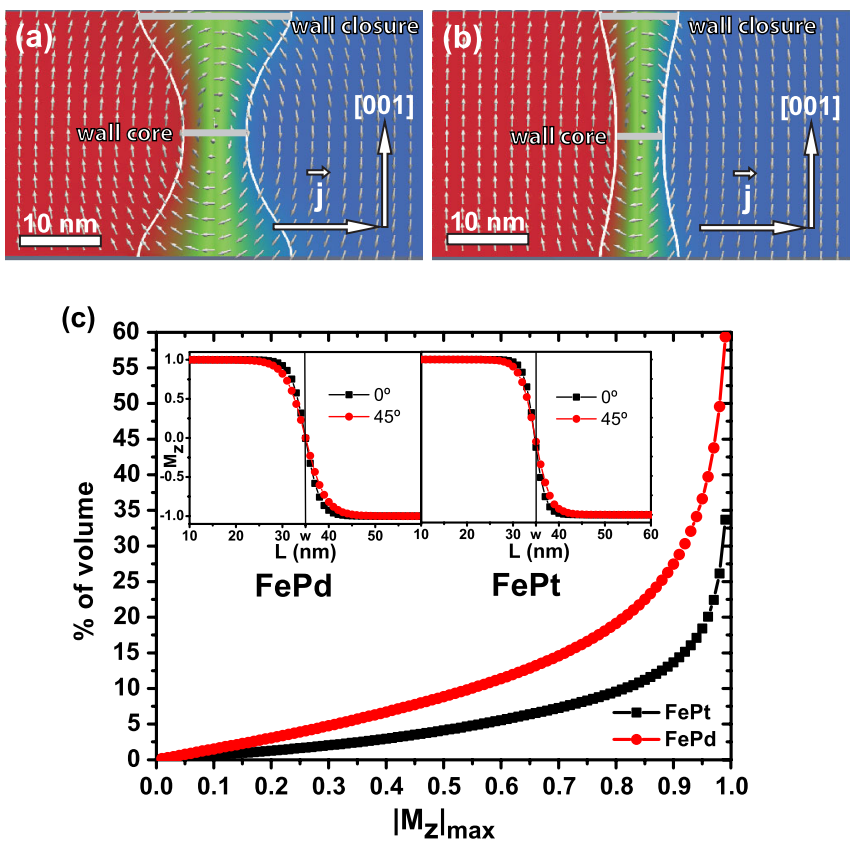

FIG. 2 (color online). Cross-sectional profiles of domain walls for (a) $L 1_{0}-\mathrm{FePd}$ and (b) $L 1_{0}$-FePt. The arrows represent the magnetization averaged over two discretization nodes within the tetrahedronal mesh. The white contour lines outline the wall at $\left|m_{z}\right|=0.75$. The directions of the current density $j$ ( $x$ axis) and the [001] axis ( $z$ axis) are pointed out in the lower right corner by arrows. The wall-closure and wall-core levels analyzed from first principles are highlighted by gray lines. The thickness of the film is $30 \mathrm{~nm}$. In (c) the volume fraction of the $M_{z}$ component of the magnetization in a disk of $600 \mathrm{~nm}$ diameter and a thickness of $30 \mathrm{~nm}$ is shown for both Fe alloys. The insets illustrate the $45^{\circ}$ profiles and the $0^{\circ}$ profiles in comparison. $w$ denotes the center of the wall. 
TABLE I. AMR and SSP contributions $\Delta R_{\mathrm{AMR}} / \rho_{0}$ and $\Delta R_{\mathrm{SSP}} / \rho_{0}$, respectively, as obtained from $a b$ initio calculations. Both the theoretical $\Delta R^{\text {theor }}$ and experimental $\Delta R^{\text {expt }}$ values are listed for comparison in units of $10^{-13} \Omega \mathrm{cm}^{2}$. The absolute interfacial resistivities are obtained considering $\rho_{0}=$ $5.18 \times 10^{-6} \Omega \mathrm{cm}$ in FePd and $\rho_{0}=13.16 \times 10^{-6} \Omega \mathrm{cm}$ in FePt.

\begin{tabular}{lcccc}
\hline \hline & $\begin{array}{c}\Delta R_{\mathrm{SSP}} / \rho_{0} \\
\left(10^{-2} \mathrm{~nm}\right)\end{array}$ & $\begin{array}{c}\Delta R_{\mathrm{AMR}} / \rho_{0} \\
\left(10^{-2} \mathrm{~nm}\right)\end{array}$ & $\Delta R^{\text {theor }}$ & $\Delta R^{\text {expt }}$ \\
\hline$L 1_{0}$-FePd & & & & 6.28 \\
Wall-core & 189.6 & -12.4 & 9.18 & \\
Wall-closure & 177.1 & -10.2 & 8.65 & \\
$L 1_{0}$-FePt & & & & 9.96 \\
Wall-core & 91.3 & -8.49 & 10.90 & \\
Wall-closure & 85.4 & -11.1 & 9.78 & \\
\hline \hline
\end{tabular}

wall volume and obtain in FePd a value of $\Delta R^{\text {expt }}=$ $6.28 \times 10^{-13} \Omega \mathrm{cm}^{2}$ and in FePt a value of $\Delta R^{\text {expt }}=$ $9.96 \times 10^{-13} \Omega \mathrm{cm}^{2}$ listed in Table I. These interfacial resistivities compare well with the experimental interfacial resistivity of $\Delta R^{\mathrm{expt}}=8.33 \times 10^{-13} \Omega \mathrm{cm}^{2}$ found in single domain wall devices of $\mathrm{FePd}$ as reported by Danneau et al. [21]. This value is nearly identical with our first-principles calculations for the spin structure determined in the closure region of the wall, discussed later, which provides for further confidence in our analysis. It is noteworthy to point out that the experimental values take into account closure region as well as the core structure of the wall which can be illustrated by a simple parallel-resistor model.

Our theoretical model of the ratio of interfacial resistivity due to a single wall, $\Delta R$, and resistivity in the absence of walls, $\rho_{0}$, is based on the assumption that it may be written in terms of a local resistivity $\rho(L)$ as

$$
\left(\frac{\Delta R}{\rho_{0}}\right)^{\text {theor }}=\int_{-\infty}^{\infty}\left(\frac{\rho(L)-\rho_{0}}{\rho_{0}}\right)^{\text {theor }} d L,
$$

where $L$ denotes the coordinate perpendicular to the wall and parallel to the $j$ axis shown in Figs. 2(a) and 2(b). The integrand in this expression is the local relative change of the resistivity due to the presence of the wall. The magnetization is characterized by its direction, $\mathbf{m}(L)$, and the $L$ derivative of it, $d \mathbf{m}(L) / d L$. Locally, the magnetic structure may be mapped onto a spin spiral with cone angle $\alpha(L)=\arcsin \left(m_{\perp}(L)\right)$ and spin-spiral vector $q(L)=$ $|d \mathbf{m}(L) / d L| / \sin (\alpha(L))$ [20]. Therefore, the local resistivity is fully characterized by $\mathbf{m}(L), q(L)$, and $\alpha(L)$, i.e., $\rho(L)=\rho(\mathbf{m}(L), \mathbf{q}(L), \alpha(L))$.

We further decompose the relative change of the magnetization into the contributions due to AMR and those due to the rotating magnetization: $\left[\rho(\mathbf{m}, \mathbf{q}, \alpha)-\rho_{0}\right] / \rho_{0}=$ $\rho_{\mathrm{AMR}}(\mathbf{m}) / \rho_{0}+\rho_{\mathrm{SSP}}(\mathbf{q}, \alpha) / \rho_{0}$. In order to compute the AMR contribution, we evaluate the resistivity $\rho_{\mathrm{C}}(\mathbf{m})$ of a collinear system as a function of $\mathbf{m}$ and set $\rho_{\mathrm{AMR}}(\mathbf{m}) / \rho_{0}=$
$\left[\rho_{\mathrm{C}}(\mathbf{m})-\rho_{\mathrm{C}}\left(\mathbf{m}_{0}\right)\right] / \rho_{\mathrm{C}}\left(\mathbf{m}_{0}\right)$, where $\mathbf{m}_{0}$ is the direction of the magnetic easy axis. Since AMR is dominantly a SOI effect, $\rho_{\mathrm{C}}(\mathbf{m})$ is evaluated from collinear calculations that include SOI. For the contribution due to the magnetization rotation in the domain wall, we set $\rho_{\mathrm{SSP}}(q, \alpha) / \rho_{0}=$ $\left[\rho_{\mathrm{NC}}(q, \alpha)-\rho_{\mathrm{NC}}(0,0)\right] / \rho_{\mathrm{NC}}(0,0)$, where the resistivity of spin spirals (SSP) with wave vector $q$ and cone angle $\alpha$, $\rho_{\mathrm{NC}}(q, \alpha)$, is obtained from noncollinear calculations for spin spirals without SOI. The relative resistivity changes $\rho_{\text {AMR }}(\mathbf{m}) / \rho_{0}$ and $\rho_{\text {SSP }}(q, \alpha) / \rho_{0}$ are calculated from $a b$ initio employing the Wannier interpolation technique [22] in conjunction with the Kubo-Greenwood formalism assuming a scalar random point defect model to describe disorder in the clean limit [20].

The AMR contribution to the interfacial domain wall resistance is computed according to

$$
\frac{\Delta R_{\mathrm{AMR}}}{\rho_{0}}=\int_{-\infty}^{\infty} \frac{\rho_{\mathrm{AMR}}(\mathbf{m}(L))}{\rho_{0}} d L,
$$

fully taking into account $\mathbf{m}(L)$ from our micromagnetic simulations. For the core region, we obtain $\Delta R_{\mathrm{AMR}} / \rho_{0}=$ $-12.4 \times 10^{-2} \mathrm{~nm}$ in $\mathrm{FePd}$ and $\Delta R_{\mathrm{AMR}} / \rho_{0}=$ $-8.49 \times 10^{-2} \mathrm{~nm}$ in FePt. Interestingly, the closure region of the wall yields very similar AMR values, shown in Table I. Noticeably, the much larger SOI strength of Pt atoms of $0.54 \mathrm{eV}$ in FePt does not result in a larger AMR contribution to the domain wall resistance, when compared to FePd with the corresponding SOI strength of $0.19 \mathrm{eV}$ for Pd atoms.

In order to calculate the SSP contribution to DW resistance, we use our micromagnetically simulated $\mathbf{m}$ profile [cf. Figs. 2(a) and 2(b)] to determine $\mathbf{q}(L)$ and $\alpha(L)$ at each point $L$ and then evaluate $\rho_{\mathrm{SSP}}(\mathbf{q}(L), \alpha(L))$ from ab initio as explained above. The absolute value of the spin-spiral wave vector $q$ is equivalent to the wavelength of the spiral $\lambda=2 \pi / q$. The calculated distribution of the inverse SSP wavelength $\lambda^{-1}$ as a function of the distance $L$ from the center of the DW is presented in the insets in Fig. 3 for the core and closure region of the DW in both materials. $\lambda^{-1}$ exhibits a rather similar dependence on $L$ at the core of the walls, in accordance to a similar core DW width for both alloys; see Fig. 2 (see also [20]). On the other hand, in correspondence to Fig. 2, the spread of $\lambda^{-1}$ with $L$ is much wider in the closure region, while $\lambda^{-1}(L)$ gives higher values in FePd than in FePt. For all considered cases, the largest $\lambda^{-1}$ observed corresponds to the smallest spinspiral pitch of $14 \mathrm{~nm}$ in the center of the DW core in $\mathrm{FePt}$, which is in good agreement with the value obtained analytically from exchange stiffness and uniaxial anisotropy constant [20].

In Fig. 3, we show the ab initio results for $\rho_{\mathrm{SSP}}(q) / \rho_{0}$ as a function of $\lambda^{-1}$. The leading order contribution to $\rho_{\mathrm{SSP}}(q) / \rho_{0}$ is proportional to $\lambda^{-1}$ even for large wavelengths, in contrast to the $\lambda^{-2}$ behavior expected from the Levy-Zhang model [16]. Similar findings for the 


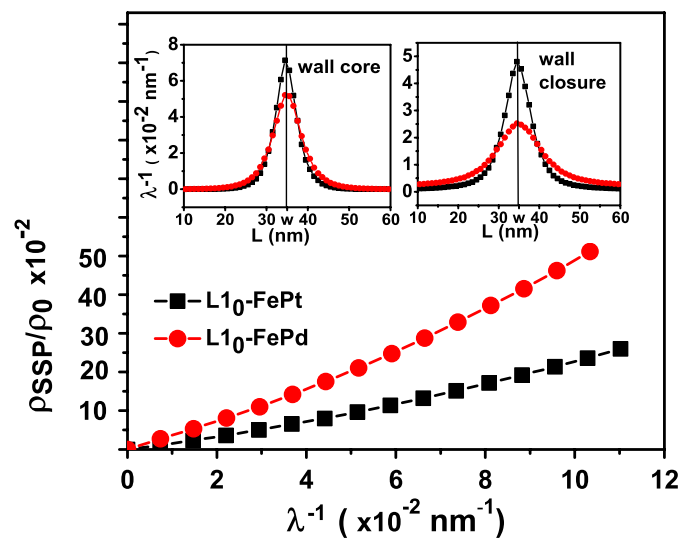

FIG. 3 (color online). Dependence of the resistivity contribution due to spin spirals of wave vector $\mathbf{q}(L)$ is shown in terms of $\rho_{\mathrm{SSP}} / \rho_{0}$ versus the inverse wavelength of the spin-spiral vectors $\lambda^{-1}$. The inset to the left illustrates the reciprocal wavelength distribution of the Bloch core at the center $w$ of the walls. The distribution of spin-spiral vectors of reciprocal wavelength $\lambda^{-1}$ is visibly narrower and yields larger values in FePt than in FePd. It decays rapidly in the closure region of the domain wall as shown in the inset to the right.

$\lambda^{-1}$ dependency in the diffuse regime have been reported earlier for supercell $a b$ initio calculations [17]. Integration according to

$$
\frac{\Delta R_{\mathrm{SSP}}}{\rho_{0}}=\int_{-\infty}^{\infty} \frac{\rho_{\mathrm{SSP}}(\mathbf{q}(L), \alpha(L))}{\rho_{0}} d L
$$

gives for the core regions $\Delta R_{\mathrm{SSP}} / \rho_{0}=1.896 \mathrm{~nm}$ in FePd and $\Delta R_{\mathrm{SSP}} / \rho_{0}=0.913 \mathrm{~nm}$ in FePt, respectively. Like for the AMR contribution, the SSP contributions of core and closure regions are similar; see Table I.

We obtain an AMR contribution to the DW resistance in FePt and FePd of not more than $10 \%$. The total $\Delta R^{\text {theor }}$ value, the sum of both AMR and SSP contributions, is in excellent agreement with $\Delta R^{\text {expt }}=9.96 \times 10^{-13} \Omega \mathrm{cm}^{2}$ measured in FePt; see Table I. In FePd the theoretical $\Delta R^{\text {theor }}$, while still smaller than in $\mathrm{FePt}$, is by some $30 \%$ larger than the experimental value of $\Delta R^{\text {expt }}=$ $6.28 \times 10^{-13} \Omega \mathrm{cm}^{2}$. This constitutes a very reasonable agreement taking into account that in the experiment the crystallographic long range order parameter was limited to $S_{\text {order }}=0.8$. Furthermore, we note that the SSP resistivity dominates over SOI-driven AMR in FePd and FePt.

In conclusion, we have analyzed the origin of the electrical resistance due to domain walls in $L 1_{0}$-FePd and $L 1_{0}$-FePt from first principles applying the KuboGreenwood formalism to the locally inhomogeneous spin structure determined from micromagnetic simulations. We found that SOI adds only a minor contribution to the total domain wall resistance in both $L 1_{0}$ alloys. Moreover, our findings indicate that the electrical resistance is clearly dominated by the contribution induced by short wavelength spin spirals. Thus, the different SOI strengths are not decisive in determining the respective DW resistances, which is contrary to the case of the anomalous Hall effect [14]. The overall resistance due to a domain wall computed in FePd and FePt agrees very well with the resistance observed in experiments.

K. M. S. thanks W. Kuch, C. H. Marrows, B. J. Hickey, A. Aziz, and M. G. Blamire and for fruitful discussions. F. G. thanks the Ministerio de Ciencia e Innovación and the Fundación Española para la Ciencia y la Tecnología of Spain for financial support. We gratefully acknowledge S. Börm and E. Westphal for discussions and assistance. F. F. and Y.M. thank the HGF-YIG program VH-NG-513 and the Jülich Supercomputing Centre for computational time.

*k.seemann@fz-juelich.de

[1] M. N. Baibich, J. M. Broto, A. Fert, F. Nguyen Van Dau, F. Petroff, P. Etienne, G. Creuzet, A. Friederich, and J. Chazelas, Phys. Rev. Lett. 61, 2472 (1988); G. Binasch, P. Grünberg, F. Saurenbach, and W. Zinn, Phys. Rev. B 39, 4828 (1989).

[2] T. Miyazaki and N. Tezuka, J. Magn. Magn. Mater. 151, 403 (1995); J. S. Moodera, L. R. Kinder, T. M. Wong, and R. Meservey, Phys. Rev. Lett. 74, 3273 (1995).

[3] J. C. Slonczewski, J. Magn. Magn. Mater. 159, L1 (1996); L. Berger, Phys. Rev. B 54, 9353 (1996).

[4] E. B. Myers, D. C. Ralph, J. A. Katine, R. N. Louie, and R. A. Buhrman, Science 285, 867 (1999).

[5] R. Lehndorff, D. E. Bürgler, S. Gliga, R. Hertel, P. Grünberg, C. M. Schneider, and Z. Celinski, Phys. Rev. B 80, 054412 (2009).

[6] S. S. P. Parkin, M. Hayashi, and L. Thomas, Science 320, 190 (2008); A. Vanhaverbeke, A. Bischof, and R. Allenspach, Phys. Rev. Lett. 101, 107202 (2008).

[7] D. A. Allwood, G. Xiong, C. C. Faulkner, D. Atkinson, D. Petit, and R. P. Cowburn, Science 309, 1688 (2005).

[8] D. Bedau, M. Kläui, S. Krzyk, U. Rüdiger, G. Faini, and L. Vila, Phys. Rev. Lett. 99, 146601 (2007).

[9] G. S. D. Beach, C. Knutson, C. Nistor, M. Tsoi, and J. L. Erskine, Phys. Rev. Lett. 97, 057203 (2006).

[10] T. A. Moore, I. M. Miron, G. Gaudin, G. Serret, S. Auffret, B. Rodmacq, A. Schuhl, S. Pizzini, J. Vogel, and M. Bonfim, Appl. Phys. Lett. 93, 262504 (2008).

[11] C. Burrowes, A. P. Mihai, D. Ravelosona, J. V. Kim, C. Chappert, L. Vila, A. Marty, Y. Samson, F. GarciaSanchez, L. D. Buda-Prejbeanu, I. Tudosa, E. E. Fullerton, and J.-P. Attane, Nature Phys. 6, 17 (2009).

[12] C. H. Marrows, Adv. Phys. 54, 585 (2005).

[13] K. M. Seemann, V. Baltz, M. MacKenzie, J. N. Chapman, B. J. Hickey, and C. H. Marrows, Phys. Rev. B 76, 174435 (2007); K. M. Seemann, M. C. Hickey, V. Baltz, B. J. Hickey, and C.H. Marrows, New J. Phys. 12, 033033 (2010).

[14] K. M. Seemann, Y. Mokrousov, A. Aziz, J. Miguel, F. Kronast, W. Kuch, M. G. Blamire, A. T. Hindmarch, B. J. Hickey, I. Souza, and C. H. Marrows, Phys. Rev. Lett. 104, 076402 (2010). 
[15] J. B. A. N. van Hoof, K. M. Schep, A. Brataas, G. E. W. Bauer, and P. J. Kelly, Phys. Rev. B 59, 138 (1999).

[16] P. M. Levy and S. Zhang, Phys. Rev. Lett. 79, 5110 (1997).

[17] B. Y. Yavorsky, I. Mertig, A. Y. Perlov, A. N. Yaresko, and V. N. Antonov, Phys. Rev. B 66, 174422 (2002).

[18] J. Stöhr, Y. Wu, B. D. Hermsmeier, M. G. Samant, G. R. Harp, S. Koranda, D. Dunham, and B. P. Tonner, Science 259, 658 (1993).

[19] R. Hertel, J. Appl. Phys. 90, 5752 (2001); A. Kákay, E. Westphal, and R. Hertel, IEEE Trans. Magn. 46, 2303 (2010).
[20] See Supplemental Material at http://link.aps.org/ supplemental/10.1103/PhysRevLett.108.077201 for details of transport experiments, micromagnetic simulations, a comparison to the analytically obtained wall widths, and more details on the Kubo-Greenwood formalism used for this work.

[21] R. Danneau, P. Warin, J.P. Attané, I. Petej, C. Beigné, C. Fermon, O. Klein, A. Marty, F. Ott, Y. Samson, and M. Viret, Phys. Rev. Lett. 88, 157201 (2002).

[22] F. Freimuth, Y. Mokrousov, D. Wortmann, S. Heinze, and S. Blügel, Phys. Rev. B 78, 035120 (2008). 